\title{
Yeast U3 localization and correct sequence (snR17a) and promotor activity (snR17b) identified by homology search
}

Sir,

snR $17 \mathrm{a}$ and $s \cap R 17 b$ from yeast, the two copy gene encoding the yeast homologue of vertebrate small nuclear RNA U3 (Hughes et al., 1987), were recently discovered to contain similar introns (Myslinski et al., 1990). Three groups (Lagosky et al., 1987; Fling et al., 1988; Barclay et al., 1988) have independently described the cloning of the dihydrofolate reductase gene (DFR1) from yeast and another (Gilbert, 1989) looked for transcription start sites on the opposite strand to the DRF1 locus. None of these groups seems to have realized from the accumulating data that they all had sequenced the same DNA region. However, a computer search for sequences homologous to the intron reveals that the $3^{\prime}$ end of $s n R 17 a$ is located exactly 311 bp upstream from the start of the open reading frame of the DFR1 gene. A contiguous sequence of $3234 \mathrm{bp}$ can be formed from all the sequences (fig. 1).

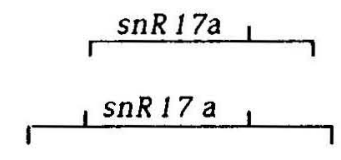

Hughes et al. (1987)

Myslinski et al. (1990)

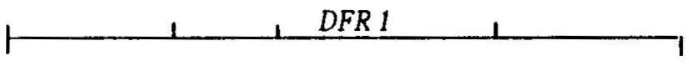

Lagosky et al.(1987)

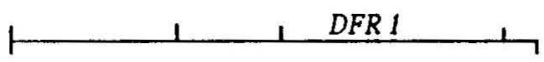

Fling et al. (1988)

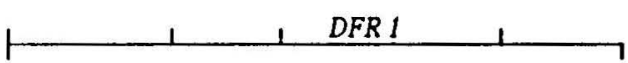

Barclay et al.(1988)

$1 \quad 1 \quad D F R \perp$

Gilbert (1989)

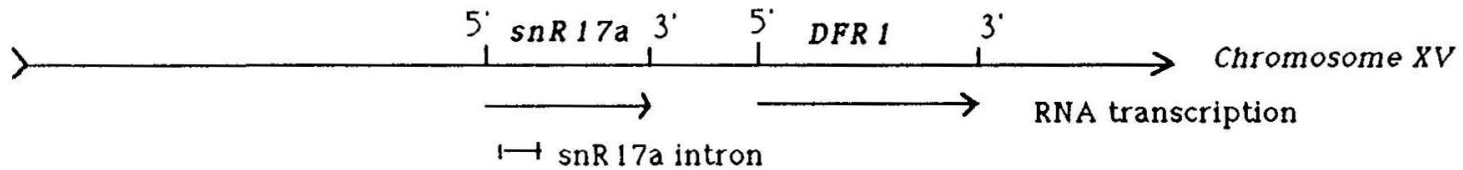

Figure 1. DNA region containing $s n R 17 \mathrm{a}$ and $D F R 1$. The regions sequenced by each of the groups are shown as lines. Start- and endpoints of the two genes on the sequenced stretches are marked. The genes already recognized by the different groups are labeled. The contiguous sequence on chromosome XV is sketched schematically at the bottom, direction and length of transcription of the respective RNAs and the position of the intron in the $s n R 17$ a gene are indicated. 
Table 1 List of the sequences containing yeast U3 found by the search

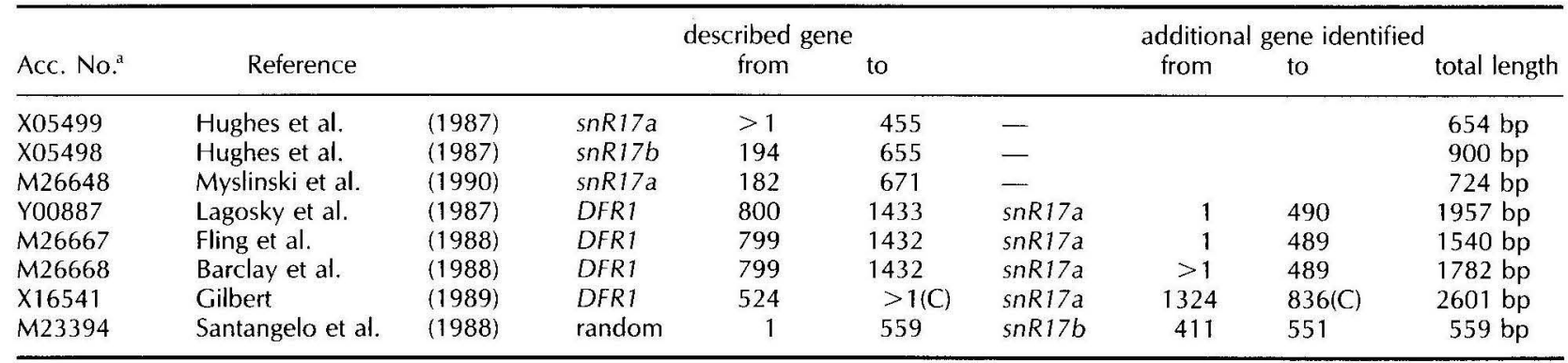

${ }^{a}$ Respective EMBL accession numbers are given in this column. The feature tables have now been updated accordingly.

The termination signals for snR 17 a partly overlap with the transcription start signals of the DFR1 gene. snR17a can now also be mapped to chromosome XV by the map position of DFR1 (Barclay et al., 1988). The four differences noted by Myslinski et al. (1990) in their $s n R 17$ a gene sequence in comparison to the data of Hughes et al. (1987) are all confirmed in the data from each of the four groups (Lagosky et al., 1987; Fling et al., 1988; Barclay et al., 1988; Gilbert, 1989) investigating the DFR1 gene. Thus the sequence of Myslinski et al. (1990) is probably the correct sequence and the folding model of the RNA encoded by the $s n R 17$ a gene (Hughes et al., 1987) has an increased hairpin 4. Apart from this, the upstream putative general amino acid control motif for DFR1 suggested by Lagosky et al. (1987) and Barclay et al. (1988) becomes less convincing as it lies within the $s n R 17 a$ RNA coding region.

A similar search for the intron from $s n R 17 b$ finds a sequence reported to be a random promotor from yeast (Santangelo et al., 1988) which turns out to be the upstream half of $s n R 17 b$ (up to six nucleotides before the $3^{\prime}$ end of the intron) plus 410 upstream nucleotides. The S1 mapping data of this group suggests that the transcription initiation site of their fusion construct lies indeed close to the revised start of the $s n R 17 b$ coding region as inferred by Myslinski et al. (1990) by the comparison of the $s n R 17 b$ gene to its RNA sequence. The related sequences are summarized in the table.

It is striking that in this case two well known genes from yeast could be sequenced repeatedly without noticing their close neighborhood and moreover, that
DNA from another copy of the first gene was considered to be random. With growing sequencing data, sequence comparisons will become more important to prevent ignoring of genes already characterized, but this will also yield valuable additional data like chromosomal location or direct evidence for promotor activity as in this case.

\section{Thomas Dandekar \\ European Molecular Biology Laboratory Postfach 10.22.09; D-69 Heidelberg Germany}

\section{REFERENCES}

Barclay, B.J., Huang, T., Nagel, M.G., Misener, V.L., Game, J.C. and Wahl, G.M. (1988) Mapping and sequencing of the dibydrofolate reductase gene (DFR 1 ) of Saccharomyces cerevisiae. Gene 63, 175-185.

Fling, M.E., Kopf, J. and Richards, C.A. (1988) Nucleotide sequence of the dihydrofolate reductase gene of Saccharomyces cerevisiae. Cene 63, 165-174.

Gilbert, S.C. (1989) Isolation of sequences upstream of the Saccharomyces cerevisiae DFR 1 gene containing a new promotor. Submitted by tape to EMBL data library, ID-code SCDFR1G.

Hughes, J.M.X., Konings, D.A.M. and Cesarini, G. (1987) The yeast homologue of U3 snRNA. EMBO 6, 2145-2155.

Lagosky, P.A., Taylor, G.R. and Haynes, R.H. (1987) Molecular characterization of the Saccharomyces cerevisiae dihydrofolate reductase gene (DFR1). Nucl. Acids Res. <15, 10355-10371.

Myslinski, E., Ségault, V. and Branlant, C. (1990) An Intron in the genes for U3 small nucleolar RNAs of the yeast Saccharomyces cerevisiae. Science 247, 1213-1216.

Santangelo, G.M., Tornow, J., McLaughlin, C.S. and Moldave, K., (1988) Properties of promotors cloned randomly from the Saccharomyces cerevisiae genome. Molecular and cellular Biology 8, 4217-4224. 